\title{
INFINITE SUMS OF ADAMS OPERATIONS AND COBORDISM
}

\author{
IMMA GÁLVEZ AND SARAH WHITEHOUSE
}

\begin{abstract}
In recent work, various algebras of stable degree zero operations in $p$-local $K$-theory were described explicitly [5]. The elements are certain infinite sums of Adams operations. Here we show how to make sense of the same expressions for $M U_{(p)}$ and $B P$, thus identifying the "Adams subalgebra" of the algebras of operations. We prove that the Adams subalgebra is the centre of the ring of degree zero operations.
\end{abstract}

\section{INTRODUCTION}

This paper builds on recent work on stable operations in $p$-local complex $K$-theory [5]. That work described all operations in terms of Adams operations. In a sense, this was done by specifying which infinite sums of Adams operations are allowed. Here we explore these expressions for $M U_{(p)}$ and $B P$.

We begin by briefly discussing the definition and basic properties of Adams operations for these theories. These facts are well known, but we hope it is helpful to gather them together here. The main results of [5] are then recalled and the analogues for $M U_{(p)}$ and $B P$ are proved, thus identifying the "Adams subalgebra" of the algebras of operations for these theories.

The final sections are devoted to showing that this subalgebra is precisely the centre of the ring of stable degree zero operations. This can be compared to old results of Novikov (for $M U$ ) and Araki (for $B P$ ), [8,2]. These state that the Adams operations form the centre of the group of stable degree zero multiplicative operations.

\section{Adams Operations}

We begin by summarizing some standard results on stable cohomology operations.

Proposition 1. [3, Section 14] Let $E=M U, B P$ or $K U$. A stable multiplicative cohomology operation $\theta: E \rightarrow E$ is uniquely determined by its value $\theta\left(x_{E}\right)$ on the orientation class $x_{E} \in E^{2}\left(\mathbb{C} P^{\infty}\right)$.

We remark that the same is true for the Adams summand of $p$-local $K$ theory, which we denote by $G$.

Date: $11^{\text {th }}$ June 2004.

2000 Mathematics Subject Classification. Primary: 55S25; Secondary: 55N22, 19 L41.

Key words and phrases. K-theory-operations - cobordism. 
In order to have stable Adams operations, we will work $p$-locally, for a prime $p$.

Definition 2. Let $E=M U_{(p)}, B P, K U_{(p)}$ or $G$. A stable Adams operation $\Psi_{E}^{\alpha}$ is defined for each $\alpha \in \mathbb{Z}_{(p)}^{\times}$as the unique multiplicative operation given on the orientation class $x_{E}$ by

$$
\Psi_{E}^{\alpha}\left(x_{E}\right)=\frac{[\alpha]_{E}\left(x_{E}\right)}{\alpha} \in E^{*}\left(\mathbb{C} P^{\infty}\right)=E_{*}\left[\left[x_{E}\right]\right],
$$

where $[\alpha]_{E}$ denotes the formal sum.

Lemma 3. $\Psi_{E}^{\alpha}$ acts as multiplication by $\alpha^{n}$ on $E_{2 n}$.

Proof. Let the log series of the formal group law corresponding to $E$ be given by $\log _{E}\left(x_{E}\right)=\sum_{i=0}^{\infty} m_{i} x_{E}^{i+1}$, where $m_{i} \in E_{2 i} \otimes \mathbb{Q}$. The operation $\Psi_{E}^{\alpha}$ transforms this into $\log _{E}\left(\left(\Psi_{E}^{\alpha}\right)^{-1}\left(x_{E}\right)\right)$, see [9, Appendix B]. This is

$$
\begin{aligned}
\log _{E}\left(\left(\Psi_{E}^{\alpha}\right)^{-1}\left(x_{E}\right)\right) & =\log _{E}\left(\exp _{E} \frac{1}{\alpha} \log _{E} \alpha x_{E}\right) \\
& =\frac{\log _{E} \alpha x_{E}}{\alpha}=\sum_{i=0}^{\infty} \alpha^{i} m_{i} x_{E}^{i+1} .
\end{aligned}
$$

Thus on coefficients, $m_{i} \mapsto \alpha^{i} m_{i}$. Since $E_{*}$ is torsion free and since $\Psi_{E}^{\alpha}$ is multiplicative, this is sufficient to determine $\Psi_{E}^{\alpha}$ on the coefficient groups.

For $E=M U, B P, K U$ or $G$, the representation of the ring of operations given by their actions on coefficient groups is faithful:

$$
E^{*}(E) \hookrightarrow \operatorname{End}\left(\mathrm{E}_{*}\right) .
$$

For $M U$ this result is due to Novikov [8, Lemma 5.2]; for $K U$, see [6, Theorem 2]. The $B P$ and $G$ versions follow. In particular, we see that Adams operations multiply as expected: $\Psi_{E}^{\alpha} \Psi_{E}^{\beta}=\Psi_{E}^{\alpha \beta}$. We also note that, since $\Psi_{E}^{\alpha}$ is multiplicative, it is a grouplike element with respect to the coproduct of $E^{*}(E), \Delta\left(\Psi_{E}^{\alpha}\right)=\Psi_{E}^{\alpha} \otimes \Psi_{E}^{\alpha}$.

If $e$ denotes the connective cover of $E$, the map $e \rightarrow E$ leads to a map $E^{*}(E) \rightarrow e^{*}(e)$. Thus we may define an Adams operation $\Psi_{e}^{\alpha}$ for $e$, with all the expected properties, as the image of $\Psi_{E}^{\alpha}$ under this map.

\section{Operations in $p$-LOCAL $K$-THEORY}

Let $K U_{(p)}$ be the $p$-local periodic complex $K$-theory spectrum and let $k u_{(p)}$ be the connective version. As above, for these theories we have stable Adams operations, $\Psi_{K U}^{\alpha}$ and $\Psi_{k u}^{\alpha}$ respectively, for each $\alpha$ in $\mathbb{Z}_{(p)}^{\times}$.

Let $p$ be an odd prime and let $q$ be primitive modulo $p^{2}$. This ensures that $q$ is primitive modulo $p^{r}$ for all $r \geq 1$, which is to say that the powers of $q$ are dense in the $p$-adic units $\mathbb{Z}_{p}^{\times}$. Clearly, the polynomial ring generated by the Adams operation $\Psi_{K U}^{q}$ is a subring of the ring of all stable degree zero 
$K U_{(p)}$ operations. This does not give all operations; it is countable and the operation algebra is known to be uncountable [1].

Definition 4. Let $p$ be an odd prime and let $q$ be primitive modulo $p^{2}$. Let $E=M U_{(p)}$ or $K U_{(p)}$ and let $e=M U_{(p)}$ or $k u_{(p)}$. We define two families of polynomials.

1) Let $\varphi_{n}^{e}=\prod_{i=0}^{n-1}\left(\Psi_{e}^{q}-q^{i}\right) \in \mathbb{Z}_{(p)}\left[\Psi_{e}^{q}\right]$.

2) Let $\Phi_{n}^{E}=\prod_{i=0}^{n-1}\left(\Psi_{E}^{q}-q_{i}\right) \in \mathbb{Z}_{(p)}\left[\Psi_{E}^{q}\right]$, where $q_{i}$ is the $i+1$-st term of the sequence

$$
1, q, q^{-1}, q^{2}, q^{-2}, q^{3}, q^{-3}, q^{4}, \ldots .
$$

For $p$ odd, the following result of [5] shows how to view the ring of all degree zero stable $K U_{(p)}$ operations as a completion of the polynomial ring $\mathbb{Z}_{(p)}\left[\Psi_{K U}^{q}\right]$. It also gives a description for the connective case, corresponding to a different completion of a polynomial ring. These results may be viewed as specifying which infinite sums of Adams operations are admissible.

Theorem 5. [5, Theorems 2.2 and 6.2]

1) Elements of $k u_{(p)}^{0}\left(k u_{(p)}\right)$ can be expressed uniquely as infinite sums

$$
\sum_{n \geq 0} a_{n} \varphi_{n}^{k u}
$$

where $a_{n} \in \mathbb{Z}_{(p)}$.

2) Elements of $K U_{(p)}^{0}\left(K U_{(p)}\right)$ can be expressed uniquely as infinite sums

$$
\sum_{n \geq 0} a_{n} \Phi_{n}^{K U}
$$

where $a_{n} \in \mathbb{Z}_{(p)}$.

Explicit product and coproduct formulas and many related results can be found in [5]. Analogues of these results hold for $p=2$, but they are more complicated. We give only the connective version.

Definition 6. Define elements $\zeta_{n}^{k u} \in k u_{(2)}^{0}\left(k u_{(2)}\right)$, for $n \geq 0$, by

$$
\begin{aligned}
\zeta_{2 m+1}^{k u} & =\left(\Psi_{k u}^{-1}-1\right) \prod_{i=0}^{m-1}\left(\Psi_{k u}^{3}-3^{2 i+1}\right), \\
\zeta_{2 m}^{k u} & =\prod_{i=0}^{m-1}\left(\Psi_{k u}^{3}-3^{2 i}\right)+\sum_{i=1}^{m} \frac{\theta_{i}(3) \theta_{i}\left(3^{2 m}\right)}{2 \theta_{i}\left(3^{2 i}\right)} \zeta_{2 m-2 i+1}^{k u},
\end{aligned}
$$

where $\theta_{r}(X)=\prod_{i=0}^{r-1}\left(X-3^{2 i}\right)$.

Theorem 7. [5, Theorem 8.2] The elements of $k u_{(2)}^{0}\left(k u_{(2)}\right)$ can be expressed uniquely as infinite sums

$$
\sum_{n \geq 0} a_{n} \zeta_{n}^{k u}
$$

where $a_{n} \in \mathbb{Z}_{(2)}$. 


\section{Infinite Sums of Adams Operations for $M U_{(p)}$}

In this section, we show that the infinite sums of Adams operations of the previous section are also defined for $M U_{(p)}$.

Proposition 8. Let $p$ be an odd prime. The infinite sums $\sum_{n=0}^{\infty} a_{n} \varphi_{n}^{M U}$, where $a_{n} \in \mathbb{Z}_{(p)}$, are well-defined operations in $M U_{(p)}$-cohomology.

Proof. Clearly, finite sums of the $\varphi_{n}^{M U}$ are well-defined operations. To see that the same is true for the infinite sums, it suffices to show that $\varphi_{n}^{M U} \rightarrow 0$ as $n \rightarrow \infty$ in the usual filtration topology of $M U_{(p)}^{*}\left(M U_{(p)}\right)$.

This may be checked by considering the action on coefficient groups. By Lemma $3, \Psi_{M U}^{q}$ acts as multiplication by $q^{i}$ on $\pi_{2 i}\left(M U_{(p)}\right)$. It follows that $\varphi_{n}^{M U}$ acts as zero on $\pi_{2 i}\left(M U_{(p)}\right)$ for $i<n$.

Proposition 9. For any odd prime p, the map

$$
k u_{(p)}^{0}\left(k u_{(p)}\right) \rightarrow M U_{(p)}^{0}\left(M U_{(p)}\right)
$$

given by

$$
\sum_{n=0}^{\infty} a_{n} \varphi_{n}^{k u} \mapsto \sum_{n=0}^{\infty} a_{n} \varphi_{n}^{M U}
$$

is an injective algebra map.

Proof. Consider $\sum_{n=0}^{\infty} a_{n} \varphi_{n}^{M U}$ in $M U_{(p)}^{0}\left(M U_{(p)}\right)$ and suppose that $a_{m} \neq 0$, with $m$ chosen minimal. This operation acts on $\pi_{2 m}\left(M U_{(p)}\right) \neq 0$ as the finite $\operatorname{sum} \sum_{n=0}^{m} a_{n} \varphi_{n}^{M U}=a_{m} \varphi_{m}^{M U}$ and thus as multiplication by $a_{m} \prod_{i=0}^{m-1}\left(q^{m}-\right.$ $\left.q^{i}\right) \neq 0$. Thus the operation is non-trivial. So the map is injective.

It is easy to see that we have an algebra map: the product of two infinite sums is determined in both the source and the target by the products of Adams operations. (See [5, Prop. 2.7] for an explicit formula.)

The source $k u_{(p)}^{0}\left(k u_{(p)}\right)$ is a completed bialgebra. We note that the injective map above also respects the coproduct, since the coproduct of a general infinite sum is determined by the fact that the Adams operations are grouplike; see [5, Prop. 2.9] for an explicit formula. So the image of $k u_{(p)}^{0}\left(k u_{(p)}\right)$ is a subbialgebra of $M U_{(p)}^{*}\left(M U_{(p)}\right)$.

There is also an injective algebra map $K U_{(p)}^{0}\left(K U_{(p)}\right) \rightarrow M U_{(p)}^{0}\left(M U_{(p)}\right)$. This is because the injection of Proposition 9 can be composed with the inclusion of bialgebras

$$
K U_{(p)}^{0}\left(K U_{(p)}\right) \hookrightarrow k u_{(p)}^{0}\left(k u_{(p)}\right),
$$

resulting from the covering map $k u_{(p)} \rightarrow K U_{(p)}$. An explicit formula for this map is given in [5, Prop. 7.1], expressing each $\Phi_{n}^{K U}$ in terms of the $\varphi_{m}^{k u}$.

Again the injection is well-behaved with respect to the coproduct. This time we have a conjugation map $\chi$ and this is also respected by the inclusion, as it is determined for both $E=K U_{(p)}$ and $E=M U_{(p)}$ by $\chi \Psi_{E}^{q}=\Psi_{E}^{q^{-1}}$. 
So far the discussion in this section has all been for odd primes. However, it is easy to see that the analogues for $p=2$ also hold. One defines $\zeta_{n}^{M U} \in$ $\mathbb{Z}_{(2)}\left[\Psi_{M U}^{3}, \Psi_{M U}^{-1}\right]$ in the obvious way, just as in Definition 6 .

Proposition 10. (1) The infinite sums $\sum_{n=0}^{\infty} a_{n} \zeta_{n}^{M U}$, where $a_{n} \in \mathbb{Z}_{(2)}$, are well-defined operations in $M U_{(2)}$-cohomology.

(2) The map $k u_{(2)}^{0}\left(k u_{(2)}\right) \rightarrow M U_{(2)}^{0}\left(M U_{(2)}\right)$ given by

$$
\sum_{n=0}^{\infty} a_{n} \zeta_{n}^{k u} \mapsto \sum_{n=0}^{\infty} a_{n} \zeta_{n}^{M U}
$$

is an injective algebra map.

Proof. The key properties of $\zeta_{n}^{k u}$ are that it acts as zero on coefficient groups $\pi_{2 i}\left(k u_{(2)}\right)$, for all $i<n$, and its action is non-zero on $\pi_{2 n}\left(k u_{(2)}\right)$. (See [5, Section 8] for a proof and related formulas.) Using this, one may check that the infinite sums are well-defined $M U_{(2)}$ operations just as in Proposition 8 and that we have the claimed injection of algebras just as in Proposition 9.

\section{Infinite Sums of Adams Operations for $B P$}

We record here the $B P$ analogues of the results of the preceding section, omitting proofs since these are easy modifications of the $M U_{(p)}$ versions.

For $p$ an odd prime, the spectra $K U_{(p)}$ and $k u_{(p)}$ each split into $p-1$ copies of spectra which we denote by $G$ and $g$, respectively ${ }^{1}$. As before we choose $q$ primitive modulo $p^{2}$ and now we let $\hat{q}=q^{p-1}$. Thus the powers of $\hat{q}$ are dense in $1+p \mathbb{Z}_{p}$. Now we recall from [5] the description of degree zero stable operations for $g$.

Definition 11. Define $\hat{\varphi}_{n}^{g} \in \mathbb{Z}_{(p)}\left[\Psi_{g}^{q}\right] \subset g^{0}(g)$, for $n \geq 0$, by

$$
\hat{\varphi}_{n}^{g}=\prod_{i=0}^{n-1}\left(\Psi_{g}^{q}-\hat{q}^{i}\right) .
$$

Also define $\hat{\varphi}_{n}^{B P} \in \mathbb{Z}_{(p)}\left[\Psi_{B P}^{q}\right] \subset B P^{0}(B P)$ in the same way.

Theorem 12. [7, Theorem 2.2], [5, Theorem 4.4] Elements of $g^{0}(g)$ can be expressed uniquely as infinite sums

$$
\sum_{n \geq 0} a_{n} \hat{\varphi}_{n}^{g}
$$

where $a_{n} \in \mathbb{Z}_{(p)}$.

Proposition 13. Let $p$ be an odd prime.

(1) The infinite sums $\sum_{n=0}^{\infty} a_{n} \hat{\varphi}_{n}^{B P}$, where $a_{n} \in \mathbb{Z}_{(p)}$, are well-defined operations in BP-cohomology.

\footnotetext{
${ }^{1}$ The notations $E(1)$ and $e(1)$ and $L$ and $l$ are also used.
} 
(2) The map $\iota: g^{0}(g) \rightarrow B P^{0}(B P)$ given by

$$
\sum_{n=0}^{\infty} a_{n} \hat{\varphi}_{n}^{g} \mapsto \sum_{n=0}^{\infty} a_{n} \hat{\varphi}_{n}^{B P}
$$

is an injective algebra map.

The same remarks as in the preceding section about the coproduct and the comparison with the periodic operations may be made here.

Now let $p=2$. Again we define $\zeta_{n}^{B P} \in \mathbb{Z}_{(2)}\left[\Psi_{B P}^{3}, \Psi_{B P}^{-1}\right]$ just as in Definition 6 .

Proposition 14. Let $p=2$.

(1) The infinite sums $\sum_{n=0}^{\infty} a_{n} \zeta_{n}^{B P}$, where $a_{n} \in \mathbb{Z}_{(2)}$, are well-defined operations in BP-cohomology.

(2) The map $\iota: k u_{(2)}^{0}\left(k u_{(2)}\right) \rightarrow B P^{0}(B P)$ given by

$$
\sum_{n=0}^{\infty} a_{n} \zeta_{n}^{k u} \mapsto \sum_{n=0}^{\infty} a_{n} \zeta_{n}^{B P}
$$

is an injective algebra map.

\section{Diagonal Operations}

We will need to discuss operations which act diagonally on coefficient groups and so we introduce the following notation.

Definition 15. Let $E=M U, M U_{(p)}$ or $B P$. We write $D_{E}$ for the subring of $E^{0}(E)$ consisting of operations whose action on each coefficient group $E_{2 i}$ is multiplication by an element $\lambda_{i}$ of the ground ring, (that is an element of $\mathbb{Z}$ for $E=M U$, an element of $\mathbb{Z}_{(p)}$ for $E=M U_{(p)}$ or $\left.E=B P\right)$.

We next recall some results from [8] on $M U$ operations. For each finite non-decreasing sequence of positive integers, $\alpha=\left(\alpha_{1}, \alpha_{2}, \alpha_{3}, \ldots\right)$, there is an operation $\bar{S}_{\alpha} \in M U^{*}(M U)$, of degree $2|\alpha|=2 \sum_{i} \alpha_{i}$. Here $\bar{S}_{\alpha}=$ $\phi\left(\bar{\sigma}_{\alpha}\right)$, where $\phi: M U^{*}(B U) \rightarrow M U^{*}(M U)$ is the Thom isomorphism, $\sigma_{\alpha} \in$ $M U^{*}(B U)$ is the Conner-Floyd characteristic class associated to $\alpha$ and $\bar{\sigma}_{\alpha}$ is defined by $\bar{\sigma}_{\alpha}(\xi)=\sigma_{\alpha}(-\xi)$.

Proposition 16. Let $E=M U, M U_{(p)}$ or BP. Then $Z\left(E^{0}(E)\right)=D_{E}$.

Proof. Clearly, $D_{E}$ is contained in the centre $Z\left(E^{0}(E)\right)$. To prove the reverse inclusion, we first let $E=M U$. Since $E_{*}$ is torsion free, an operation $\theta$ is zero if and only if $\theta_{*} \otimes 1_{\mathbb{Q}}$ is zero on $E_{*} \otimes \mathbb{Q}=\mathbb{Q}\left[\mathbb{C} P^{1}, \mathbb{C} P^{2}, \ldots\right]$. So we can work with monomials in the classes $\left[\mathbb{C} P^{n}\right]$. We first consider the operation $\bar{S}_{(m)} \in E^{2 m}(E)$. We let $\mathcal{S}_{(m)}=\left[\mathbb{C} P^{m}\right] \bar{S}_{(m)} \in E^{0}(E)$. This operation has the property that it acts as zero on any decomposable class of degree $2 m$ and as multiplication by $(m+1)$ on $\left[\mathbb{C} P^{m}\right]$. Let $\theta \in Z\left(E^{0}(E)\right)$. The relation $\mathcal{S}_{(m)} \theta=\theta \mathcal{S}_{(m)}$ then tells us that $\theta_{*}\left[\mathbb{C} P^{m}\right]=\lambda_{m}\left[\mathbb{C} P^{m}\right]$ for some $\lambda_{m} \in \mathbb{Z}$.

More generally, for $\alpha=\left(\alpha_{1}, \alpha_{2}, \ldots, \alpha_{r}\right)$, we have a Landweber-Novikov operation $\bar{S}_{\alpha} \in E^{2|\alpha|}(E)$. By $\left[8\right.$, Lemma 5.5], $\left(\bar{S}_{\alpha}\right)_{*}\left(\left[\mathbb{C} P^{n}\right]\right)=\lambda_{\alpha}\left[\mathbb{C} P^{n-|\alpha|}\right]$, 
where $\lambda_{\alpha} \in \mathbb{Z}$ and $\lambda_{\alpha} \neq 0$. Let $\mathbb{C} P^{\alpha}=\mathbb{C} P^{\alpha_{1}} \times \mathbb{C} P^{\alpha_{2}} \times \cdots \times \mathbb{C} P^{\alpha_{r}}$ and let $\mathcal{S}_{\alpha}=\left[\mathbb{C} P^{\alpha}\right] \bar{S}_{\alpha} \in E^{0}(E)$. Then the relation $\left(\mathcal{S}_{\alpha} \theta\right)_{*}\left[\mathbb{C} P^{|\alpha|}\right]=\left(\theta \mathcal{S}_{\alpha}\right)_{*}\left[\mathbb{C} P^{|\alpha|}\right]$ tells us that $\theta$ acts on $\left[\mathbb{C} P^{\alpha}\right]$ as multiplication by $\lambda_{|\alpha|} \in \mathbb{Z}$. Thus $\theta \in D_{E}$.

The argument is just as above for $M U_{(p)}$ and that for $B P$ is similar, but here we need only work with monomials in the $\left[\mathbb{C} P^{p^{i}-1}\right]$, since $B P_{*} \otimes \mathbb{Q}=$ $\mathbb{Q}\left[\mathbb{C} P^{p^{i}-1} \mid i \geq 1\right]$.

We remark that, if one considers the algebra of all operations, rather than only degree zero ones, then the centre consists of just the constant operations.

\section{Congruences}

In this section $p$ is an odd prime and we compare two families of congruences, one related to the connective Adams summand $g$ and the other to $B P$. The results will be used in the next section to give a new description of the centre of $B P^{0}(B P)$.

Clearly, we may identify the ring of diagonal operations $D_{B P}$ with a subring of the infinite direct product $\prod_{k \geq 0} \mathbb{Z}_{(p)}$. In what follows we will often use this identification without further comment.

We begin by noting the analogue for the connective Adams summand $g$ of [4, Theorem 11]. This characterizes $g^{0}(g)$ as a subring of the infinite direct product $\prod_{k \geq 0} \mathbb{Z}_{(p)}$ by a family of congruences which involve Gaussian polynomials.

Definition 17. The Gaussian polynomial $\left[\begin{array}{c}n \\ i\end{array}\right] \in \mathbb{Z}[t]$ is defined, for nonnegative integers $n$ and $i$, by

$$
\left[\begin{array}{l}
n \\
i
\end{array}\right]=\prod_{k=0}^{i-1} \frac{1-t^{n-k}}{1-t^{i-k}} .
$$

Also let $\left[\begin{array}{c}n \\ i\end{array}\right]_{a}$ denote the value of this polynomial at $t=a$.

Theorem 18. If $\phi \in g^{0}(g)$ acts on $g_{2(p-1) i}=\mathbb{Z}_{(p)}$ as multiplication by $\mu_{i} \in \mathbb{Z}_{(p)}$, then

$$
\sum_{i=0}^{n}(-1)^{n-i} \hat{q}^{\left(\begin{array}{c}
n-i \\
2
\end{array}\right)}\left[\begin{array}{c}
n \\
i
\end{array}\right]_{\hat{q}} \mu_{i} \equiv 0 \quad \bmod p^{\delta_{p}(n)},
$$

for all $n \geq 0$, where $\delta_{p}(n)=n+\nu_{p}(n !)$. Moreover every sequence satisfying these congruences arises from a unique stable operation.

Proof. A $\mathbb{Z}_{(p)}$-basis for $G_{0}(g)$ is given in [5, Prop. 4.2]. Using [4, Prop. 8] this may be expressed in terms of Gaussian polynomials. The result follows from the fact that $g^{0}(g)$ is the $\mathbb{Z}_{(p)}$-linear dual of $G_{0}(g)$.

Definition 19. Let

$$
C_{n, i}=\frac{(-1)^{n-i} \hat{q}^{\left(\begin{array}{c}
n-i \\
2
\end{array}\right)}\left[\begin{array}{l}
n \\
i
\end{array}\right]_{\hat{q}}}{p^{\delta_{p}(n)}} \in \mathbb{Q}
$$

and let $C_{n}=\left(C_{n, i}\right)_{i \geq 0}$, a sequence of rational numbers. 
We note that $C_{n, n}=p^{-\delta_{p}(n)}$ and $C_{n, i}=0$ when $i>n$. Also note that $\delta_{p}\left(p^{r}\right)=1+p+p^{2}+\cdots+p^{r}$. The $n$th congruence of Theorem 18 may be expressed as $\sum_{i=0}^{n} C_{n, i} \mu_{i} \in \mathbb{Z}_{(p)}$ or, adopting vector notation, $C_{n} \cdot \mu \in \mathbb{Z}_{(p)}$.

The rest of this section will be devoted to comparing the congruences of Theorem 18 with a system of congruences characterizing $D_{B P}$.

Next we recall that, using duality, an operation $\theta: B P \rightarrow B P$ is determined by the left $B P_{*}$-module map $\bar{\theta}=\langle\theta\rangle:, B P_{*}(B P) \rightarrow B P_{*}$, where $\langle$,$\rangle is the Kronecker pairing. The action of the operation on coefficient$ groups $\theta_{*}: B P_{*} \rightarrow B P_{*}$ is obtained by composition with the right unit map: $\theta_{*}=\bar{\theta} \eta_{R}$. (See, for example, $[3,11.22]$.)

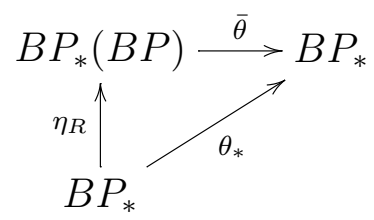

We will adopt the $B P$ notation of $[9]$. Thus $B P_{*}=\mathbb{Z}_{(p)}\left[v_{1}, v_{2}, \ldots\right]$, where the $v_{i}$ are Araki's generators, $\left|v_{i}\right|=2 p^{i}-2$, and $B P_{*}(B P)=B P_{*}\left[t_{1}, t_{2}, \ldots\right]$, where $\left|t_{i}\right|=2 p^{i}-2$. We also use the elements $l_{i} \in B P_{*} \otimes \mathbb{Q}$, related recursively to the $v_{i}$ by the formula of Araki. As usual, because all objects are torsion free, one may calculate rationally. We will do this implicitly, so that, for example, we write $\bar{\theta}\left(l_{i}\right)$ to mean $\left(\bar{\theta} \otimes 1_{\mathbb{Q}}\right)\left(l_{i}\right)$.

We suppose that we have $\theta \in D_{B P}$, acting on $B P_{2(p-1) i}$ as multiplication by $\mu_{i} \in \mathbb{Z}_{(p)}$. (So, in the notation of Sect. $6, \mu=\left(\lambda_{0}, \lambda_{p-1}, \lambda_{2(p-1)}, \ldots\right)$.) By consideration of the relation $\theta_{*}=\bar{\theta} \eta_{R}$, one may calculate recursively $\bar{\theta}(x)$ for $x \in B P_{*}(B P)$ in terms of the sequence $\mu=\left(\mu_{i}\right)_{i \geq 0}$. Since, in $\bar{\theta}(x)$, the coefficient of each monomial in the generators $v_{i}$ must lie in $\mathbb{Z}_{(p)}$, one finds congruences imposed on the terms of this sequence.

We now give more details of this system of congruences. Let $\alpha, \beta, \gamma, \delta, \epsilon$ be finite sequences of non-negative integers. If $\alpha=\left(\alpha_{1}, \alpha_{2}, \ldots, \alpha_{m}\right)$ then $v^{\alpha}$ denotes the monomial $v_{1}^{\alpha_{1}} v_{2}^{\alpha_{2}} \ldots v_{m}^{\alpha_{m}}$ and let $\|\alpha\|=\sum_{i=1}^{m} \alpha_{i}(1+p+\cdots+$ $\left.p^{i-1}\right)=\frac{\left|v^{\alpha}\right|}{2(p-1)}$.

Let $E_{\beta, \gamma}^{\alpha} \in \mathbb{Z}_{(p)}$ be determined by $\eta_{R}\left(v^{\alpha}\right)=\sum_{\beta, \gamma} E_{\beta, \gamma}^{\alpha} v^{\beta} t^{\gamma}$. Then, since $\bar{\theta} \eta_{R}\left(v^{\alpha}\right)=\theta_{*}\left(v^{\alpha}\right)$, we find that

$$
\sum_{\beta+\delta=\epsilon} \sum_{\gamma} E_{\beta, \gamma}^{\alpha} D_{\delta}^{\gamma}(\mu)= \begin{cases}\mu_{\|\alpha\|} & \text { if } \alpha=\epsilon \\ 0 & \text { otherwise }\end{cases}
$$

where $\bar{\theta}\left(t^{\gamma}\right)=\sum_{\delta} D_{\delta}^{\gamma}(\mu) v^{\delta}$. Now (20) determines $D_{\delta}^{\gamma}(\mu)$ recursively as a finite rational linear combination of the $\mu_{i}$. On the other hand, we have $D_{\delta}^{\gamma}(\mu) \in \mathbb{Z}_{(p)}$, for all $\gamma$ and $\delta$, and these integrality conditions form our family of congruences.

For example, $D_{(1)}^{(1)}(\mu)=a\left(\frac{\mu_{1}-\mu_{0}}{p}\right)$, where $a \in \mathbb{Z}_{(p)}^{\times}$. So $\frac{\mu_{1}-\mu_{0}}{p} \in \mathbb{Z}_{(p)}$. Notice that this is the congruence $C_{1} \cdot \mu \in \mathbb{Z}_{(p)}$ of Theorem 18 . 
In order to compare the congruences of Theorem 18 and the $B P$ ones, we need some further notation.

Definition 21. Let $\mu$ denote an element in $\prod_{k \geq 0} \mathbb{Z}_{(p)}$ and define the following subsets of $\prod_{k \geq 0} \mathbb{Z}_{(p)}$.

$$
\begin{aligned}
S_{\infty}^{g} & =\left\{\mu \mid C_{i} \cdot \mu \in \mathbb{Z}_{(p)} \text { for all } 0 \leq i\right\}, \\
S_{n}^{g} & =\left\{\mu \mid C_{i} \cdot \mu \in \mathbb{Z}_{(p)} \text { for } 0 \leq i \leq n\right\}, \\
S_{\infty}^{B P} & =\left\{\mu \mid D_{\delta}^{\gamma}(\mu) \in \mathbb{Z}_{(p)} \text { for all } \gamma, \delta\right\}, \\
S_{n}^{B P} & =\left\{\mu \mid \exists \mu^{\prime} \in S_{\infty}^{B P} \text { with } \mu_{i}=\mu_{i}^{\prime} \text { for } 0 \leq i \leq n\right\} .
\end{aligned}
$$

Thus $g^{0}(g) \cong S_{\infty}^{g}$ by Theorem 18 and $D_{B P} \cong S_{\infty}^{B P}$ by the preceding discussion. Also, clearly

$$
\begin{gathered}
S_{0}^{g} \supset S_{1}^{g} \supset \cdots \supset S_{n}^{g} \supset S_{n+1}^{g} \supset \cdots \supset S_{\infty}^{g}=\bigcap_{n=0}^{\infty} S_{n}^{g}, \\
S_{0}^{B P} \supset S_{1}^{B P} \supset \cdots \supset S_{n}^{B P} \supset S_{n+1}^{B P} \supset \cdots \supset S_{\infty}^{B P}=\bigcap_{n=0}^{\infty} S_{n}^{B P} .
\end{gathered}
$$

Lemma 22. For all $n \geq 0, S_{n}^{g} \subseteq S_{n}^{B P}$.

Proof. Let $\mu \in S_{n}^{g}$. Given the form of the congruences of Theorem 18, it is easy to see that there is some $\mu^{\prime} \in S_{\infty}^{g} \cong g^{0}(g)$ such that $\mu_{i}=\mu_{i}^{\prime}$ for $0 \leq i \leq n$. Then, using the injection $\iota$ of Proposition 13, we can find an operation $\theta \in B P^{0}(B P)$ inducing $\mu^{\prime}$. Thus $\mu^{\prime} \in S_{\infty}^{B P}$. But then $\mu \in S_{n}^{B P}$.

In due course, we will establish the reverse inclusion, using induction on $n$. Note that the congruence $C_{n} \cdot \mu \in \mathbb{Z}_{(p)}$ has the form

$$
p^{-\delta_{p}(n)}\left(\mu_{n}+y\right) \in \mathbb{Z}_{(p)},
$$

where $y$ is a $\mathbb{Z}_{(p)}$ linear combination of $\mu_{n-1}, \ldots, \mu_{0}$. We will see that it is enough to find a $B P$ congruence like this for each $n$. Although the following lemma is rather elementary, for convenience we record the precise formulation we need, leaving the proof to the reader.

Lemma 23. For each $r \geq 0$, let $c_{r}=\left(c_{r, i}\right)_{i \geq 0}$ be a sequence of rational numbers such that

$$
\begin{aligned}
c_{r, i} & \in p^{-\delta_{p}(r)} \mathbb{Z}_{(p)}, \quad \text { for } 0 \leq i \leq r-1, \\
c_{r, r} & \in p^{-\delta_{p}(r)} \mathbb{Z}_{(p)}^{\times}, \\
c_{r, i} & =0, \quad \text { for } i>r .
\end{aligned}
$$

Let $\widehat{c}_{r}$ be another sequence satisfying the same conditions as $c_{r}$. 
Let $n \geq 0$ and let

$$
\begin{aligned}
& S_{n}=\left\{\mu \in \prod_{k \geq 0} \mathbb{Z}_{(p)} \mid c_{r} \cdot \mu \in \mathbb{Z}_{(p)} \text { for } 0 \leq r \leq n\right\}, \\
& T_{n}=\left\{\mu \in \prod_{k \geq 0} \mathbb{Z}_{(p)} \mid c_{r} \cdot \mu \in \mathbb{Z}_{(p)} \text { for } 0 \leq r \leq n-1, \widehat{c}_{n} \cdot \mu \in \mathbb{Z}_{(p)}\right\} .
\end{aligned}
$$

If $S_{n} \subseteq T_{n}$ then $S_{n}=T_{n}$.

Of course, the $C_{r}$ of Definition 19 satisfy the hypotheses of the $c_{r}$ in the lemma.

Definition 24. Let $\theta \in D_{B P}$, with $\theta_{*}=\mu \in S_{\infty}^{B P}$. Define $V_{\mu}: B P_{*}(B P) \rightarrow$ $\mathbb{Z}_{(p)}$ as the composite $V_{\mu}=\pi \bar{\theta}$, where $\pi: B P_{*} \rightarrow \mathbb{Z}_{(p)}$ is the algebra map determined by $\pi\left(v_{1}\right)=1$ and $\pi\left(v_{i}\right)=0$ for all $i>1$.

Thus, for $x \in B P_{*}(B P), V_{\mu}(x)$ is a finite rational linear combination of the $\mu_{i}$ and the fact that it lies in $\mathbb{Z}_{(p)}$ is one of our $B P$ congruences. We will see that it is enough to consider only those $B P$ congruences arising in this way.

Lemma 25. Let $x, y \in B P_{*}(B P)$. Suppose $V_{\mu}(x)=\sum_{i=0}^{r} a_{i} \mu_{i}$ and $V_{\mu}(y)=$ $\sum_{j=0}^{s} b_{j} \mu_{j}$. Then $V_{\mu}(x y)=\sum_{i=0}^{r} \sum_{j=0}^{s} a_{i} b_{j} \mu_{i+j}$.

Proof. This is easy to check, using that $\eta_{R}$ is a ring homomorphism, that $\bar{\theta}$ is a left $B P_{*}$-module homomorphism and that $\bar{\theta} \eta_{R}=\theta_{*}=\mu$.

We recall the standard notation $\pi_{n}=p-p^{p^{n}}$. We write, for $n \geq 1$, $\bar{\pi}_{n}=\frac{\pi_{n}}{p}=1-p^{p^{n}-1} \in \mathbb{Z}_{(p)}^{\times}$and $\bar{\alpha}_{n}=\prod_{i=1}^{n} \bar{\pi}_{i} \in \mathbb{Z}_{(p)}^{\times}$.

Lemma 26. $V_{\mu}(1)=\mu_{0}$ and for $i \geq 0$,

$$
V_{\mu}\left(t_{i+1}\right)=p^{-(i+1)} \bar{\alpha}_{i+1}^{-1} \mu_{\delta_{p}\left(p^{i}\right)}-\sum_{k=1}^{i+1} p^{-k} \bar{\alpha}_{k}^{-1} V_{\mu}\left(t_{i+1-k}^{p^{k}}\right) .
$$

Proof. It is easily checked using induction and the Araki formula that, for $k \geq$ 1 , the coefficient of $v_{1}^{\delta_{p}\left(p^{k-1}\right)}$ in $l_{k}$ is $p^{-k} \bar{\alpha}_{k}^{-1} \in \mathbb{Q}$. We recall that $\eta_{R}\left(l_{i+1}\right)=$ $\sum_{k=0}^{i+1} l_{k} t_{i+1-k}^{p^{k}}$ and thus

$$
\mu_{\delta_{p}\left(p^{i}\right)} l_{i+1}=\theta_{*}\left(l_{i+1}\right)=\bar{\theta} \eta_{R}\left(l_{i+1}\right)=\sum_{k=0}^{i+1} l_{k} \bar{\theta}\left(t_{i+1-k}^{p^{k}}\right) .
$$

Now (27) follows by equating coefficients of $v_{1}^{\delta_{p}\left(p^{i}\right)}$.

Now we produce our special $B P$ congruence.

Proposition 28. For each $n \geq 0$, there is an element $d_{n} \in B P_{*}(B P)$ such that $V_{\mu}\left(d_{n}\right)=\sum_{j=0}^{n} d_{n, j} \mu_{j}$, where $d_{n, j} \in p^{-\delta_{p}(n)} \mathbb{Z}_{(p)}$ for $0 \leq j \leq n-1$ and $d_{n, n} \in p^{-\delta_{p}(n)} \mathbb{Z}_{(p)}^{\times}$. 
Proof. Firstly, we note that it is enough to prove this for $n=p^{i}$ for each $i \geq 0$. For suppose that we have found $d_{p^{i}}$, for $i \geq 0$, then, writing $N=\sum_{k=0}^{M} a_{k} p^{k}$, where $0 \leq a_{k} \leq p-1$, it is straightforward to check using Lemma 25 that we may put $d_{N}=\prod_{k=0}^{M} d_{p^{k}}^{a_{k}}$.

Now we will construct $d_{p^{i}}$ inductively. We adopt the induction hypothesis that such an element exists and moreover can be chosen of the form $t_{i+1}+$ $p r_{i+1}$, for some $r_{i+1} \in B P_{*}(B P)$. For $i=0$, we may take $d_{1}=t_{1}$, since $V_{\mu}\left(t_{1}\right)=p^{-1} \bar{\alpha}_{1}^{-1}\left(\mu_{1}-\mu_{0}\right)$. Now we assume that we have constructed $d_{p^{j}}$ for $0 \leq j<i$ and we explain how to construct $d_{p^{i}}$.

For the remainder of this proof, for $a$ and $b$ finite rational linear combinations of the $\mu_{k}$, we write $a \sim b$ to mean that $a$ and $b$ differ by some element of the form $a_{0} \mu_{0}+\cdots+a_{p^{i}} \mu_{p^{i}}$, where $a_{k} \in p^{-\delta_{p}\left(p^{i}\right)} \mathbb{Z}_{(p)}$ for $0 \leq k \leq p^{i}-1$ and $a_{p^{i}} \in p^{-\delta_{p}\left(p^{i}\right)+1} \mathbb{Z}_{(p)}$. Note that, if $a \sim b$ and if $a$ satisfies the conditions required for $V_{\mu}\left(d_{p^{i}}\right)$, then so does $b$.

Using Lemma 25, it is not hard to see that we can correct for the first term on the right-hand side of the equation (27). In fact, we can find $\bar{r}_{0} \in$ $B P_{*}(B P)$, a $\mathbb{Z}_{(p)}$ linear combination of $d_{1}^{\delta_{p}\left(p^{i}\right)}, \ldots, d_{1}^{p^{i}+1}$, such that

$$
V_{\mu}\left(t_{i+1}-p \bar{r}_{0}\right) \sim-\sum_{k=1}^{i} p^{-k} \bar{\alpha}_{k}^{-1} V_{\mu}\left(t_{i+1-k}^{p^{k}}\right) .
$$

Let $1 \leq k \leq i$. By our induction hypothesis, $d_{p^{i-k}}=t_{i-k+1}+p r_{i-k+1}$, for some $r_{i-k+1} \in B P_{*}(B P)$. It follows that

$$
d_{p^{i-k}}^{p^{k}}=\left(t_{i-k+1}+p r_{i-k+1}\right)^{p^{k}}=t_{i-k+1}^{p^{k}}+p^{k+1} \bar{r}_{i-k+1},
$$

for some $\bar{r}_{i-k+1} \in B P_{*}(B P)$.

Now we set

$$
d_{p^{i}}=t_{i+1}-p \bar{r}_{0}-p \sum_{k=1}^{i} \bar{\alpha}_{k}^{-1} \bar{r}_{i-k+1} \in B P_{*}(B P) .
$$

Clearly $d_{p^{i}}$ has the form required by the induction hypothesis. Using (29) and (30), a calculation shows that

$$
V_{\mu}\left(d_{p^{i}}\right) \sim-\sum_{k=1}^{i} p^{-k} \bar{\alpha}_{k}^{-1} V_{\mu}\left(d_{p^{i-k}}^{p^{k}}\right) .
$$

Now, using the induction hypothesis and Lemma 25, one checks that $V_{\mu}\left(d_{p^{i}}\right)$ has the required form.

Now fix a choice of $d_{n} \in B P_{*}(B P)$ as in Proposition 28 and let $C_{n}^{B P}=$ $\left(d_{n, i}\right)_{i \geq 0}$. Then the $B P$ congruence $V_{\mu}\left(d_{n}\right) \in \mathbb{Z}_{(p)}$ may be written $C_{n}^{B P} \cdot \mu \in$ $\mathbb{Z}_{(p)}$.

Proposition 31. For all $n \geq 0, S_{n}^{B P}=S_{n}^{g}$. 
Proof. This will be proved by induction on $n$. It is true for $n=0$, since $S_{0}^{B P}=S_{0}^{g}=\prod_{k \geq 0} \mathbb{Z}_{(p)}$. Now we assume that $S_{n-1}^{B P}=S_{n-1}^{g}$. Let

$$
T_{n}^{B P}=\left\{\mu \in \prod_{k \geq 0} \mathbb{Z}_{(p)} \mid C_{r} \cdot \mu \in \mathbb{Z}_{(p)} \text { for } 0 \leq r \leq n-1, C_{n}^{B P} \cdot \mu \in \mathbb{Z}_{(p)}\right\} .
$$

By Lemma 22, $S_{n}^{g} \subseteq S_{n}^{B P}$. Now if $\mu \in S_{n}^{B P}$, then $\mu \in S_{n-1}^{B P}=S_{n-1}^{g}$, by the induction hypothesis, and so $C_{r} \cdot \mu \in \mathbb{Z}_{(p)}$ for $0 \leq r \leq n-1$. Also, if $\mu \in S_{n}^{B P}$, there is $\mu^{\prime} \in S_{\infty}^{B P}$ such that $\mu_{i}=\mu_{i}^{\prime}$ for $0 \leq i \leq n$. By Proposition 28, $C_{n}^{B P} \cdot \mu^{\prime} \in \mathbb{Z}_{(p)}$. But $C_{n}^{B P} \cdot \mu^{\prime}=C_{n}^{B P} \cdot \mu$. So $S_{n}^{B \bar{P}} \subseteq T_{n}^{B P}$. Thus we have

$$
S_{n}^{g} \subseteq S_{n}^{B P} \subseteq T_{n}^{B P}
$$

Applying Lemma 23 with $c_{r}=C_{r}$ and $\widehat{c}_{r}=C_{r}^{B P}$ gives $S_{n}^{g}=T_{n}^{B P}$. So $S_{n}^{B P}=S_{n}^{g}$.

\section{The Centre of the Ring of Degree Zero Operations}

Definition 32. (1) We define the Adams subalgebra of $M U_{(p)}$ operations, $A_{M U_{(p)}}$, by

$$
\begin{aligned}
& A_{M U_{(p)}}=\left\{\sum_{n=0}^{\infty} a_{n} \varphi_{n}^{M U} \mid a_{n} \in \mathbb{Z}_{(p)}\right\} \subset M U_{(p)}^{0}\left(M U_{(p)}\right), \quad \text { for } p \text { odd }, \\
& A_{M U_{(2)}}=\left\{\sum_{n=0}^{\infty} a_{n} \zeta_{n}^{M U} \mid a_{n} \in \mathbb{Z}_{(2)}\right\} \subset M U_{(2)}^{0}\left(M U_{(2)}\right) .
\end{aligned}
$$

(2) We define the Adams subalgebra of $B P$ operations, $A_{B P}$, by

$$
\begin{aligned}
& A_{B P}=\left\{\sum_{n=0}^{\infty} a_{n} \hat{\varphi}_{n}^{B P} \mid a_{n} \in \mathbb{Z}_{(p)}\right\} \subset B P^{0}(B P), \quad \text { for } p \text { odd }, \\
& A_{B P}=\left\{\sum_{n=0}^{\infty} a_{n} \zeta_{n}^{B P} \mid a_{n} \in \mathbb{Z}_{(2)}\right\} \subset B P^{0}(B P), \quad \text { for } p=2 .
\end{aligned}
$$

Each Adams subalgebra is the image of an injective algebra map constructed in Section 4 or 5 and it follows that there are isomorphisms:

$$
\begin{aligned}
& A_{M U_{(p)}} \cong k u_{(p)}^{0}\left(k u_{(p)}\right), \quad A_{B P} \cong g^{0}(g), \quad \text { for } p \text { odd }, \\
& A_{M U_{(2)}} \cong k u_{(2)}^{0}\left(k u_{(2)}\right), \quad A_{B P} \cong k u_{(2)}^{0}\left(k u_{(2)}\right), \quad \text { for } p=2 \text {. }
\end{aligned}
$$

In Section 6, we identified the centre of the ring of all degree zero stable operations with the ring of diagonal operations. In this section we show that this is precisely the Adams subalgebra. Clearly, the Adams subalgebra is contained in the diagonal operations; we need to see that this inclusion is in fact an equality. We begin with $B P$ at odd primes, since this case follows directly from the results of the previous section. 
Theorem 33. Let $p$ be an odd prime. The centre of the ring of degree zero $B P$ operations is the Adams subalgebra:

$$
Z\left(B P^{0}(B P)\right)=A_{B P}
$$

Proof. By Proposition 16, the centre is equal to the ring of diagonal operations, so it is enough to show that $D_{B P}=A_{B P}$. By Proposition 13 and Theorem $18, A_{B P} \cong g^{0}(g) \cong S_{\infty}^{g}$. Also $D_{B P} \cong S_{\infty}^{B P}$. Here the isomorphisms are given by sending operations to their actions on coefficients. By Proposition $31, S_{n}^{B P}=S_{n}^{g}$ for all $n$ and so $S_{\infty}^{B P}=S_{\infty}^{g}$. So we have a commutative diagram

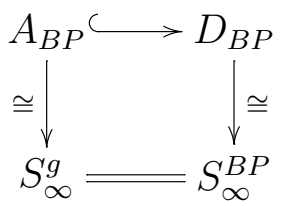

and thus $D_{B P}=A_{B P}$.

Now we need to explain how to prove the corresponding result for $B P$ when $p=2$ and for $M U_{(p)}$ for all primes. The ideas involved are the same as those for $B P$ at odd primes. Therefore we explain how the arguments need to be modified, without giving all the details.

We begin by noting that, as in [6] and [4], we can characterize $k u^{0}(k u)$ and $k u_{(p)}^{0}\left(k u_{(p)}\right)$ by systems of congruences. Thus, letting an operation in $k u^{0}(k u)$ act on $\pi_{2 i}(k u)$ as multiplication by $\lambda_{i} \in \mathbb{Z}$, we have

$$
k u^{0}(k u) \cong S_{\infty}^{k u}:=\left\{\lambda \in \prod_{k \geq 0} \mathbb{Z} \mid \sum_{j=0}^{i} \frac{v_{i, j}}{d(i)} \lambda_{j} \in \mathbb{Z} \text { for all } i \geq 0\right\} .
$$

Here $d(i)=\prod_{p \text { prime }} p^{\gamma_{p}(i)}$, where $\gamma_{p}(i)=\delta_{p}(\lfloor i /(p-1)\rfloor)$. Descriptions of the integers $v_{i, j}$ can be found in [6] and [4]. (More precisely, these references give algorithms for the $v_{i, j}$, but not a global formula.) We also have

$$
k u_{(p)}^{0}\left(k u_{(p)}\right) \cong S_{\infty}^{k u_{(p)}}:=\left\{\lambda \in \prod_{k \geq 0} \mathbb{Z}_{(p)} \mid \sum_{j=0}^{i} \frac{v_{i, j}}{d(i)} \lambda_{j} \in \mathbb{Z}_{(p)} \text { for all } i \geq 0\right\} .
$$

Using duality and [4, Props. 3, 20], it is possible to write an equivalent system of $p$-local $k u$ congruences explicitly, in terms of Gaussian polynomials, just as we did in the previous section for the Adams summand $g$. However, for our purposes here, it is enough to note that we have one congruence for each non-negative integer $n$ and this takes the form $p^{-\gamma_{p}(n)}\left(\lambda_{n}+y\right) \in \mathbb{Z}_{(p)}$, where $y$ is a $\mathbb{Z}_{(p)}$ linear combination of $\lambda_{0}, \lambda_{1}, \ldots, \lambda_{n-1}$. Of course, we may also define $S_{n}^{k u}$ and $S_{n}^{k u_{(p)}}$ in the obvious way, by imposing the relevant congruences for $0 \leq i \leq n$.

Theorem 34. Let $p=2$. The centre of the ring of degree zero BP operations is the Adams subalgebra:

$$
Z\left(B P^{0}(B P)\right)=A_{B P}
$$


Proof. As before, the centre is the ring of diagonal operations, so we need to show that $A_{B P}=D_{B P}$. Using Proposition $14, A_{B P} \cong k u_{(2)}^{0}\left(k u_{(2)}\right) \cong S_{\infty}^{k u_{(2)}}$. Again $D_{B P} \cong S_{\infty}^{B P}$. For each $n \geq 0$, the inclusion $S_{n}^{k u_{(2)}} \subseteq S_{n}^{B P}$ follows from Proposition 14. To establish the reverse inclusions, it is necessary to produce a suitable $B P$ congruence for each non-negative integer $n$, of the form described above for the congruences defining $S_{\infty}^{k u_{(2)}}$. Noting that, for $p=2, \gamma_{p}(n)=\delta_{p}(n)$, we see that such a congruence is provided by Proposition 28 (which does hold for $p=2$ ). Just as in the previous section, we conclude that $S_{n}^{B P}=S_{n}^{k u_{(2)}}$ for all $n \geq 0$, and therefore $S_{\infty}^{B P}=S_{\infty}^{k u_{(2)}}$. The result follows.

Now we turn to the results for $M U_{(p)}$. We have $M U_{*}=\mathbb{Z}\left[x_{1}, x_{2}, \ldots\right]$, where $\left|x_{i}\right|=2 i$, and $M U_{*}(M U)=M U_{*}\left[b_{1}, b_{2}, \ldots\right]$, where $\left|b_{i}\right|=2 i$. Now let $\theta \in D_{M U}$ act on $\pi_{2 i}(M U)$ as multiplication by $\lambda_{i} \in \mathbb{Z}$. Just as for $B P$, we can identify $D_{M U}$ with a set of sequences characterized by a family of congruences. Thus we set $\bar{\theta}\left(b^{\gamma}\right)=\sum_{\delta} \Delta_{\delta}^{\gamma}(\lambda) x^{\delta}$, and the $\Delta_{\delta}^{\gamma}(\lambda)$ are determined recursively as finite rational linear combinations of the $\lambda_{i}$ by $\bar{\theta} \eta_{R}=\theta_{*}=\lambda$. Then we define

$$
S_{\infty}^{M U}=\left\{\lambda \in \prod_{k \geq 0} \mathbb{Z} \mid \Delta_{\delta}^{\gamma}(\lambda) \in \mathbb{Z} \text { for all } \gamma, \delta\right\},
$$

and we have $D_{M U} \cong S_{\infty}^{M U}$. Similarly, we let

$$
S_{\infty}^{M U_{(p)}}=\left\{\lambda \in \prod_{k \geq 0} \mathbb{Z}_{(p)} \mid \Delta_{\delta}^{\gamma}(\lambda) \in \mathbb{Z}_{(p)} \text { for all } \gamma, \delta\right\},
$$

and we see that $D_{M U_{(p)}} \cong S_{\infty}^{M U_{(p)}}$.

Theorem 35. For any prime $p$, the centre of the ring of degree zero $M U_{(p)}$ operations is the Adams subalgebra:

$$
Z\left(M U_{(p)}^{0}\left(M U_{(p)}\right)\right)=A_{M U_{(p)}} .
$$

Proof. By Proposition 16, the centre is the ring of diagonal operations and we need to show that $A_{M U_{(p)}}=D_{M U_{(p)}}$. Using Propositions 9 and 10, for all primes $p$, we have

$$
A_{M U_{(p)}} \cong k u_{(p)}^{0}\left(k u_{(p)}\right) \cong S_{\infty}^{k u_{(p)}}
$$

Also, $D_{M U_{(p)}} \cong S_{\infty}^{M U_{(p)}}$. Again the isomorphisms are given by sending operations to their actions on coefficients. So we need to show that $S_{\infty}^{M U_{(p)}}=S_{\infty}^{k u_{(p)}}$. We will explain how to exploit the results for $B P$ to show this.

Suppose that $\theta \in D_{M U_{(p)}}$ acts on $\pi_{2 n}\left(M U_{(p)}\right)$ as multiplication by $\lambda_{n}$. Using the same arguments as for $B P$, it will be enough to find a particular family of $M U_{(p)}$ congruences satisfied by the $\lambda_{i}$. Specifically, we need, for each $n \geq 0$, an $M U_{(p)}$ congruence of the form $p^{-\gamma_{p}(n)}\left(\lambda_{n}+y\right) \in \mathbb{Z}_{(p)}$, where 
$y$ is a $\mathbb{Z}_{(p)}$ linear combination of $\lambda_{0}, \lambda_{1}, \ldots, \lambda_{n-1}$. This is because $S_{\infty}^{k u_{(p)}}$ is given by such a system of congruences, as explained above.

Now $M U_{(p)_{*}}\left(M U_{(p)}\right)$ is a polynomial extension of $B P_{*}(B P)$ and we write $i$ : $B P_{*}(B P) \hookrightarrow M U_{(p)_{*}}\left(M U_{(p)}\right)$ for the inclusion. The elements $d_{k}$ of $B P_{*}(B P)$, constructed in Proposition 28, give us elements $i\left(d_{k}\right)$ in $M U_{(p)_{*}}\left(M U_{(p)}\right)$. Let $V_{\lambda}: M U_{(p)_{*}}\left(M U_{(p)}\right) \rightarrow \mathbb{Z}_{(p)}$ be given by composing $\bar{\theta}: M U_{(p)_{*}}\left(M U_{(p)}\right) \rightarrow$ $M U_{(p)_{*}}$ with the algebra map $M U_{(p)_{*}} \rightarrow \mathbb{Z}_{(p)}$ which sends $x_{p-1}$ to 1 and $x_{j}$ to zero for all $j \neq p-1$. Then, for $n=k(p-1)$ we can deduce the required congruence easily from the corresponding $B P$ one, using $V_{\lambda}\left(i\left(d_{k}\right)\right)=$ $V_{\mu}\left(d_{k}\right) \in \mathbb{Z}_{(p)}$, where $\mu=\left(\lambda_{0}, \lambda_{p-1}, \lambda_{2(p-1)}, \ldots\right)$.

For $p=2$, this gives all the required congruences. Finally, let $p$ be an odd prime and let $n=k(p-1)+j$, with $0 \leq j \leq p-2$. Since $\gamma_{p}(n)=\delta_{p}(k)$, we need a congruence of the form $p^{-\delta_{p}(k)}\left(\lambda_{n}+y\right) \in \mathbb{Z}_{(p)}$, where $y$ is some $\mathbb{Z}_{(p)}$ linear combination of $\lambda_{0}, \ldots, \lambda_{n-1}$. By definition of $V_{\lambda}$, the sum over $r$ of the coefficients of $x_{p-1}^{r}$ in $\bar{\theta}\left(i\left(d_{k}\right)\right)$ is $V_{\lambda}\left(i\left(d_{k}\right)\right)$. Now we have $\bar{\theta}\left(2 b_{1}\right)=\left(\lambda_{1}-\lambda_{0}\right) x_{1}$ and it follows that $\bar{\theta}\left(2^{j} b_{1}^{j}\right)=\left(\sum_{l=0}^{j}(-1)^{j-l}\left(\begin{array}{l}j \\ l\end{array}\right) \lambda_{l}\right) x_{1}^{j}$. Using this, it may be checked that a congruence of the required type is provided by the sum over $r$ of the coefficients of the terms $x_{1}^{j} x_{p-1}^{r}$ in $\bar{\theta}\left(2^{j} b_{1}^{j} i\left(d_{k}\right)\right)$.

We end with a result about integral $M U$.

Theorem 36. $Z\left(M U^{0}(M U)\right) \cong k u^{0}(k u)$.

Proof. By Proposition 16, $Z\left(M U^{0}(M U)\right)=D_{M U}$. Also $D_{M U} \cong S_{\infty}^{M U}$ and $k u^{0}(k u) \cong S_{\infty}^{k u}$. Now we have

$$
S_{\infty}^{M U}=\bigcap_{p} S_{\infty}^{M U_{(p)}} \quad \text { and } \quad S_{\infty}^{k u}=\bigcap_{p} S_{\infty}^{k u_{(p)}}
$$

The proof of Theorem 35 shows that, for every prime $p, S_{\infty}^{M U_{(p)}}=S_{\infty}^{k u_{(p)}}$ and so $S_{\infty}^{M U}=S_{\infty}^{k u}$.

\section{REFERENCES}

[1] J. F. Adams and F. W. Clarke, Stable operations on complex K-theory, Illinois J. Math. 21 (1977), 826-829.

[2] S. Araki, Multiplicative operations in BP cohomology, Osaka J. Math. 12 (1975), $343-356$.

[3] J. M. Boardman, Stable operations in generalized cohomology. Handbook of algebraic topology. Edited by I. M. James. (North-Holland, Amsterdam 1995), 585-686.

[4] F. Clarke, M. D. Crossley and S. Whitehouse, Bases for cooperations in K-Theory, $K$-theory 23 (2001), 237-250.

[5] F. Clarke, M. Crossley and S. Whitehouse, Algebras of operations in K-theory, to appear in Topology, arxiv:math.KT/0401414.

[6] K. Johnson, The action of the stable operations of complex K-theory on coefficient groups, Illinois J. Math. 28 (1984), 57-63.

[7] W. Lellmann, Operations and co-operations in odd-primary connective $K$-theory, J. London Math. Soc. 29 (1984), 562-576.

[8] S. P. Novikov, The methods of algebraic topology from the viewpoint of cobordism theory, Math. USSR-Izv. 1 (1967), 827-913. 
[9] D. C. Ravenel, Nilpotence and periodicity in stable homotopy theory, Annals of Mathematics Studies No. 128. (Princeton University Press, Princeton NJ 1992).

Computing, Communications Technology and Mathematics, London MetROPOLITAN University, Holloway RoAd, LONDON N7 8DB, UK.

E-mail address: i.galvezicarrillo@londonmet.ac.uk

Pure Mathematics, University of Sheffield, Sheffield S3 7RH, UK.

E-mail address: s.whitehouse@sheffield.ac.uk 